\title{
A Circuit Model for Antenna Array Mutual Coupling Effects
}

Kun-Chou Lee and *Tah-Hsiung Chu

Department of Electrical Engineering, National Taiwan University

Taipei, Taiwan, Republic of China

\section{Introduction}

In this paper, a circuit model is developed to represent the input admittance of an antenna array with finite elements. It consists of a component to represent the input admittance of an isolate antenna element and an infinite shunt components with each to represent different degrees of antenna mutual coupling effects. This model is shown not only to be numerically efficient comparing to the moment method analysis, but also to give physical insight into the antenna array mutual coupling mechanism.

\section{Circuit Model}

Consider a finite antenna array of $N$-dipoles, with each element excited by a voltage $E_{i}$ respectively. The resulting feed currents $\bar{I}$, and excitation voltages $\bar{E}$ are related by $\overline{\bar{Z}} \bar{I}=\bar{E}$. If all the array elements are identical, the $\overline{\bar{Z}}$ matrix can be written as $Z_{i s o}(\overline{\bar{U}}+\overline{\bar{M}})$, where $Z_{\text {iso }}$ is the self-impedance of an isolate antenna element, $\overline{\bar{U}}$ is a unit matrix, and $\overline{\bar{M}}$ is a matrix to represent the antenna array mutual coupling effects with its elements given by

$$
M_{i j}= \begin{cases}0 & \text { for } i=j \\ Z_{i j} / Z_{i s o} & \text { otherwise. }\end{cases}
$$

By applying the bionomial expansion to $\overline{\bar{Z}}^{-1}$, we have

$$
\bar{I}=\frac{1}{Z_{\text {iso }}}\left(\overline{\bar{U}}-\overline{\bar{M}}+\overline{\bar{M}}^{2}-\overline{\bar{M}}^{3}+\cdots\right) \bar{E} .
$$

Based on the above formulation, the input admittance $Y_{i n}^{i}$ of the $i$-th antenna element can be expressed as

$$
\begin{aligned}
Y_{i n}^{i} & =\frac{I_{i}}{E_{i}} \\
& =Y_{i s o}+Y_{i}^{(1)}+Y_{i}^{(2)}+Y_{i}^{(3)}+\cdots,
\end{aligned}
$$

where

$$
Y_{i s o}=\frac{1}{Z_{i s o}}
$$




$$
\begin{aligned}
Y_{i}^{(1)} & =-\frac{Y_{i s o}}{E_{i}}\left(\sum_{\substack{j \\
j \neq i}} M_{i j} E_{j}\right) \\
Y_{i}^{(2)} & =\frac{Y_{i s o}}{E_{i}}\left(\sum_{\substack{j \\
j \neq i}} \sum_{\substack{k \\
k \neq j}} M_{i j} M_{j k} E_{k}\right) \\
Y_{i}^{(3)} & =-\frac{Y_{i s o}}{E_{i}}\left(\sum_{\substack{j \\
j \neq i}} \sum_{\substack{k \\
k \neq j}} \sum_{\substack{l \\
l \neq k}} M_{i j} M_{j k} M_{k l} E_{l}\right) .
\end{aligned}
$$

Therefore, a circuit model composed of a component of self-admittance term $Y_{i s o}$ in shunt with an infinite components $Y_{i}^{(n)}$ can be established as shown in Fig.1 to represent the $i$-th element input admittance $Y_{i n}^{i}$.

Physically, the $Y_{\text {iso }}$ component of the model is due to the contribution of the antenna excitation $E_{i}$ only. The $Y_{i}^{(1)}$ is the first-order mutual coupling term, which is due to the direct coupling effects from the other $N-1$ dipoles to the $i$-th antenna element. The term $M_{i j}$ in (5) means the direct coupling on the $i$-th dipole from the $j$-th dipole. Similarly, $Y_{i}^{(2)}$ component is the secondorder coupling and the term $M_{i j} M_{j k}$ in (6) means the "two-trip" coupling from the $k$-th dipole to the $j$-th dipole then to the $i$-th dipole, as shown in Fig.2. Other shunt components $Y_{i}^{(3)}, Y_{i}^{(4)}, \cdots$, can be interpreted in the same manner.

\section{Numerical Example}

In the numerical study, the array elements are equally spaced by $0.75 \lambda$ in both $\hat{y}$ - and $\hat{z}$-directions, as shown in Fig.3(a). The resulting error of each antenna element input admittance is given as

$$
\text { Error }=\left|\frac{\left|Y_{\text {modect }}\right|-\left|Y_{\text {exact }}\right|}{\left|Y_{\text {esact }}\right|}\right| \times 100 \% \text {. }
$$

The $Y_{\text {exact }}$ in (8) is the exact input admittance which is solved by Pocklington equation using moment method and Galerkin procedure [1][2], while $Y_{\text {model }}$ is the input admittance calculated using the circuit model.

First, if the mutual coupling effects are completely ignored, $Y_{\text {model }}$ is identical to the $Y_{i s o}$. The resulting percentage error distribution of array elements is shown in Fig.3(b) about $21 \%$ to $45 \%$, and the root mean square error of array admittance is $37.4 \%$. In order to identify the antenna array mutual coupling effect, we first apply the circuit model to include only $Y_{i s o}$ and $Y_{i}^{(1)}$. The resulting percentage error is shown reduced to about between $5.4 \%$ and $26.5 \%$ as given in Fig.3(c), and the root mean square error of array input admittance is $13.3 \%$. Finally, Fig.3(d) shows that the error of input admittance of most elements can be reduced below $7 \%$, and the root mean square error is about 
$4.23 \%$ by including the second-order shunt component $Y_{i}^{(2)}$. More accurate results can be achieved as the higher order shunt components considered.

\section{Conclusion}

In this paper, a circuit model is developed to represent the input admittance of antenna array with finite elements. Physically, this model is equivalent to the Twersky's treatment of multiple scattering [3]. Since the array computation does not involve matrix inversion, it is suitable for large array analysis which the exact antenna array theory is numerically difficult. Furthermore, this model has no limitation on antenna array geometry and excitation.

\section{References}

[1] R. F. Harrington, Field Computation by Moment Methods, New York: Macmillan, 1968.

[2] W. L. Stutzman and G. A. Thiele, Antenna Theory and Design, New York: John Wiley \& Sons, 1981.

[3] V. Twersky, "Multiple scattering of radiation by arbitrary configuration of parallel cylinders," J. Acoust. Soc. Amer., vol. 24, No. 1, pp. 42-46, Jan. 1952.

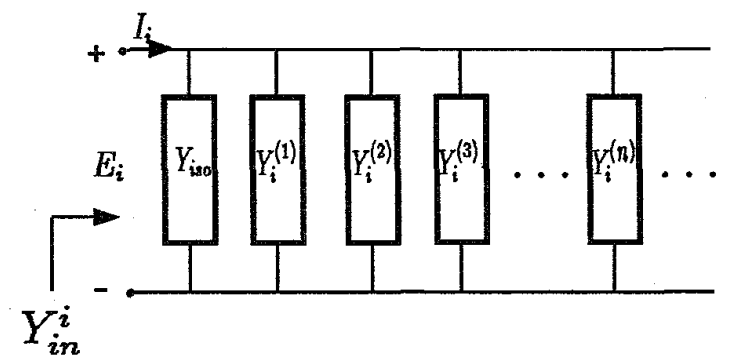

Fig. 1 Circuit model for the $i$-th antenna element input admittance.

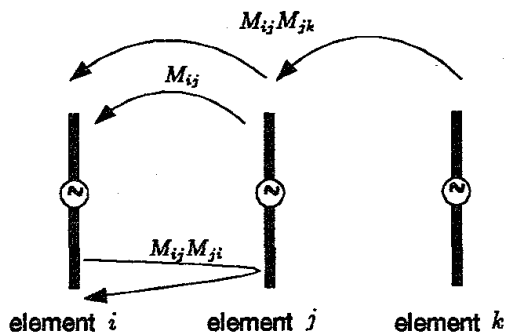

Fig. 2 Interpretation of mutual coupling mechanism. 

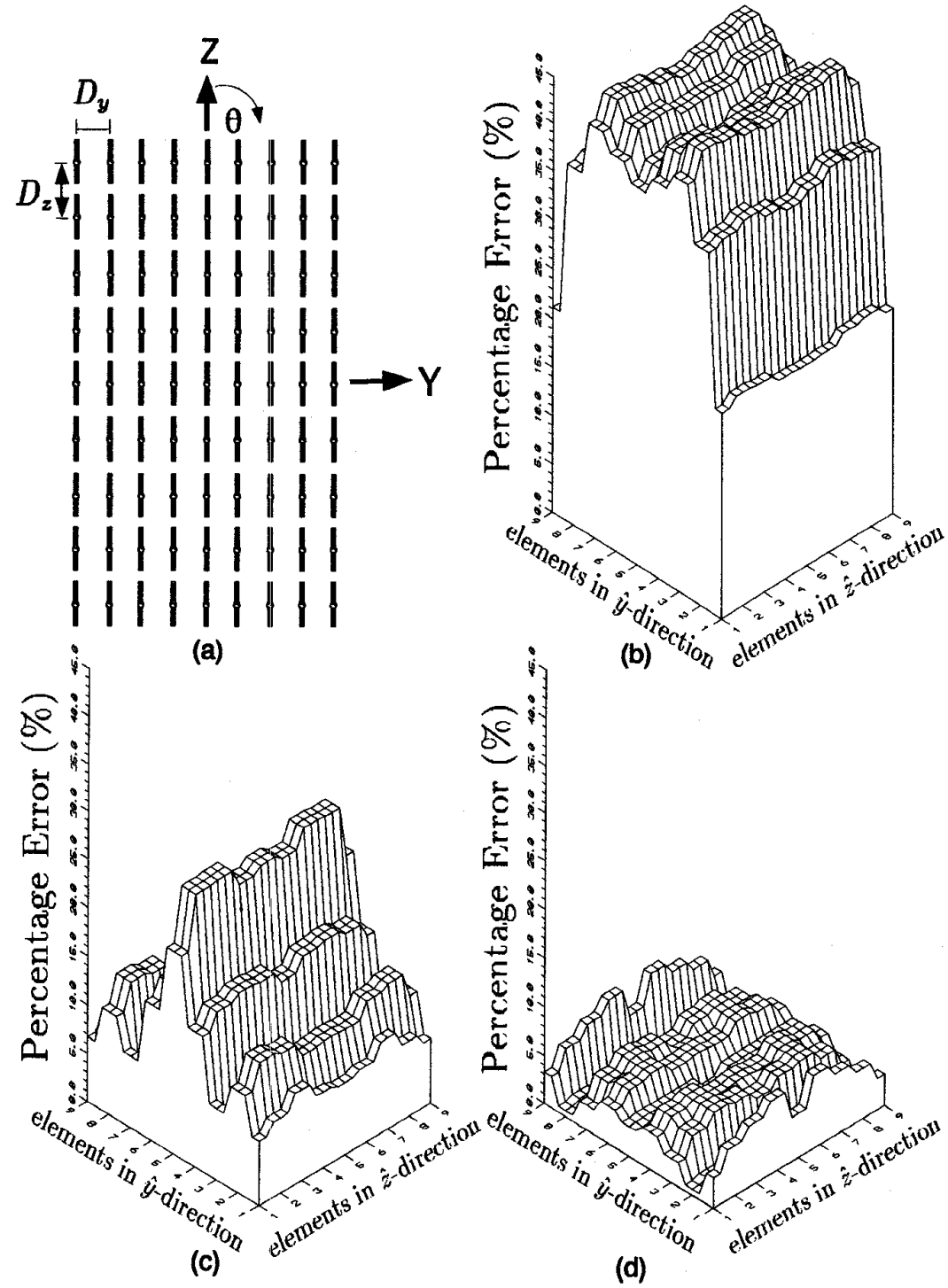

Fig. 3 (a) Geometry of a $9 \times 9$ dipolc array, and the percentage crror distribution of input admittancs by using circuit model including (b) sclf-admittance $Y_{i s o}$ only, (c) $Y_{i s o}$ and mutual-admittance tecm $Y_{i}^{(1)}$, and (d) $Y_{i s o}$ and mutual-admittance terms $Y_{i}^{(1)}$ and $Y_{i}^{(2)}$. 